\title{
Chapter 6. Significance of the urine test
}

(C) Japanese Society of Nephrology 2009

- The urine test (proteinuria and/or hematuria) is a simple and efficient method for the detection of CKD.

- Proteinuric patients constitute a high-risk group for ESKD and CVD.

- Risk for progression toward ESKD is higher in proportion to the amount of urinary protein excretion and high when urine is positive for both proteinuria and hematuria.

- Examination of microalbuminuria is useful for early detection of diabetic nephropathy.

- Since the presence of proteinuria is a sign for poor prognosis, the urine test is necessary in CVD patients.

- Among the markers for kidney damage, urine abnormality, especially proteinuria, is the most important. Particularly in early stage CKD without obvious manifestations (such as chronic glomerulonephritis), the urine test is the only measure for its early detection and is simple, inexpensive and accurate.

- In Japan, the School Health Law requires every school child (in elementary school), pupil (in middle and high school), student (in college) and teacher to undergo urine testing. Urine testing is required for all working people as well as the general population aged 40 years or older by The Industrial Safety and Health Law and the Law of Health and Medical Services for the Aged. Together, these three laws comprise a health checkup system providing lifetime urine testing. We are privileged to have such an ideal screening system in terms of early detection, prevention and education for kidney disease.
- Chronic glomerulonephritis is decreasing as a cause of ESKD. This may be attributed to early detection and treatment through the mandatory urine checkup system in Japan.

- In the urine protein test by dipstick, the incidence of proteinuria is as low as $0.5 \%$, but the possibility of these subjects entering dialysis is as high as 5-10\%. About $3 \%$ of subjects with both proteinuria and hematuria have had to have dialysis therapy within 10 years. There was no difference in the cumulative incidence of dialysis between cases with hematuria alone (mostly in elderly women) and those without proteinuria or hematuria. The cumulative incidence of dialysis was $16 \%$ for $3+$ or greater and about $7 \%$ for $2+$ of proteinuria by dipstick during 17-year follow-up. These results suggest that the risk of developing ESKD is proportional to the degree of proteinuria (Fig. 6-1).

- The risk of developing cardiovascular disease (CVD) increases with the reduction of kidney function, and it becomes even higher when proteinuria is present (Fig. 6-2). The American Heart Association (AHA), therefore, recommends the urine test for CVD patients, because proteinuria is considered to be an important risk factor for CVD progression.

- Recently, with the prevalence of obesity and unhealthy lifestyles at younger ages, the incidence of abnormal urine tests is increasing. This justifies urine testing in school-age children.

- Chronic glomerulonephritis, such as IgA nephropathy, often detected by health checkups in Japan, can be successfully treated by intensive therapy, including the early use of corticosteroid or immunosuppressive 


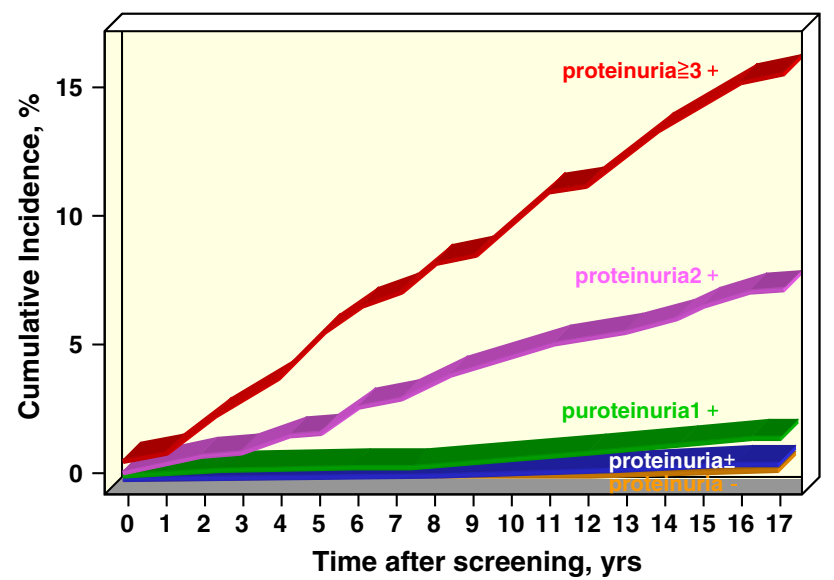

Fig. 6-1 Cumulative incidence of ESKD in CKD patients with different degrees of proteinuria. The data are quoted, with modification, from Iseki K et al. (Kidney Int. 2003;63:1468-1474)

agents. Thus, early detection and treatment are very important.

- The earliest marker for diabetic nephropathy is microalbuminuria, which can be alleviated or normalized by

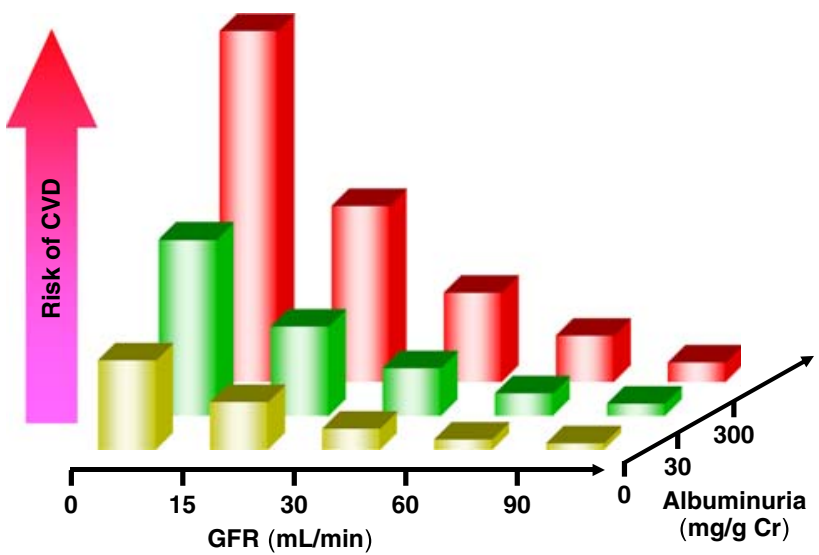

Fig. 6-2 Declining GFR and increasing proteinuria as independent and additional risk factors. The data are quoted, with modification, from K/DOQI Clinical Practice Guidelines [Am. J. Kidney Dis. 2004;43(Suppl 1):S1-S290]

ACE inhibitors and/or ARBs and by strict control of blood glucose. 